\title{
XIV.
}

\section{Leber-Cirrhose und multiple Adenom-Bildung der Leber.}

(Aus dem Pathologischen Institut der Universität Göttingen.)

Von

Dr. V. Schmieden,

z. Z. Volontär-Assistenten des Instituts.

(Hierzu Tafel VIII.)

Die Untersuchungen über die Entstehung der Leber-Adenome, ihre Abgrenzung gegenüber andersartigen Störungen und ihre Classification sind ein keineswegs abgeschlossenes Gebiet, und ehe das letzte Wort über sie gesprochen werden kann, wird noch eine grosse Reihe von genauen Untersuchungen und Beschreibungen erforderlich sein. Die genane makroskopische und mikroskopische Beschreibung eines jeden in dieses Gebiet gehörenden Falles scheint mir um so wichtiger zu sein, als die Fälle von ächtem Leberadenom als sehr seltene zu bezeiehnen sind, besonders aber, weil die exacte Untersuchung noch in jedem der bisher veröffentlichten Fälle höchst interessante, theilweise unerwartete Befunde, insbesondere in der Zell- und Kernstructur geliefert hat, Befunde, die im Einzelnen so mannigfaltig sind, dass in der ganzen Literatur kaum zwei Fälle zu finden sein dürften, die sich völlig in Parallele mit einander setzen liessen. Die Fragen, wo hört die einfache Hypertrophie auf, wo beginnt die Adenom-Bildung, und wo ist die Grenze des Adenoms gegen den Krebs? was ist der Mutterboden der Neubildung? was ist die Veranlassung zur Entstehung? das sind die Fragen, die bei den Untersuchungen immer im Mittelpunkte des Interesses standen. Bei der grossen Verschiedenheit im Bau der bisher beobachteten Leber-Adenome und bei der vielfachen Deutung, die sie erfuhren, ist es nicht zu verwundern, dass ein jeder Autor eine gesonderte Ansicht über sie hat, und dass die Ver- 
Fig. 7
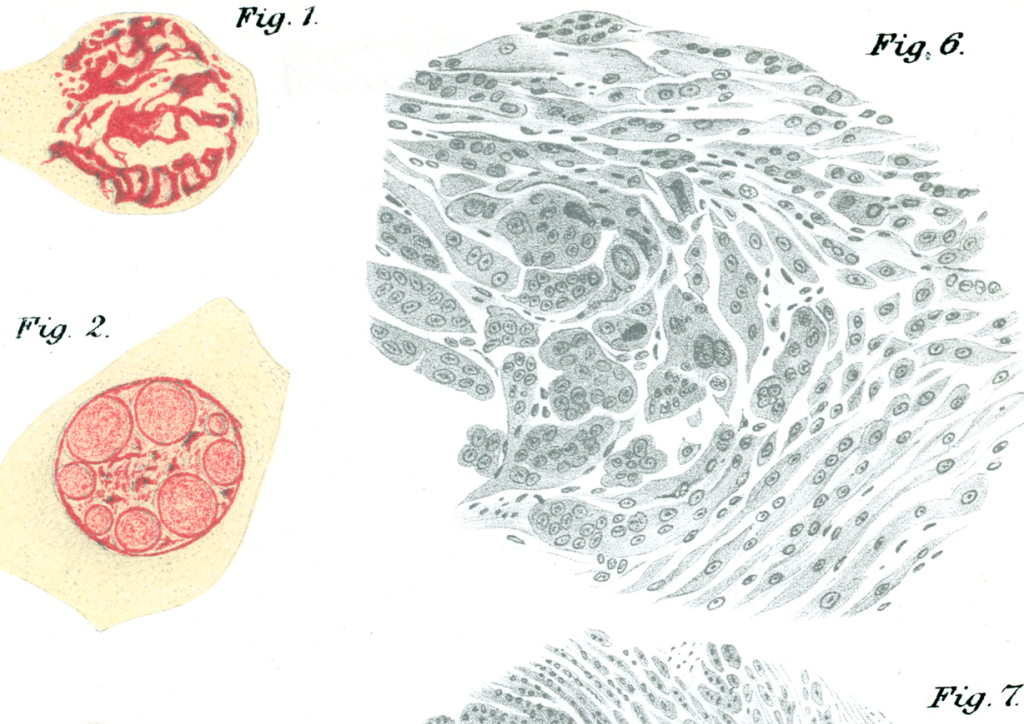

Fig.3
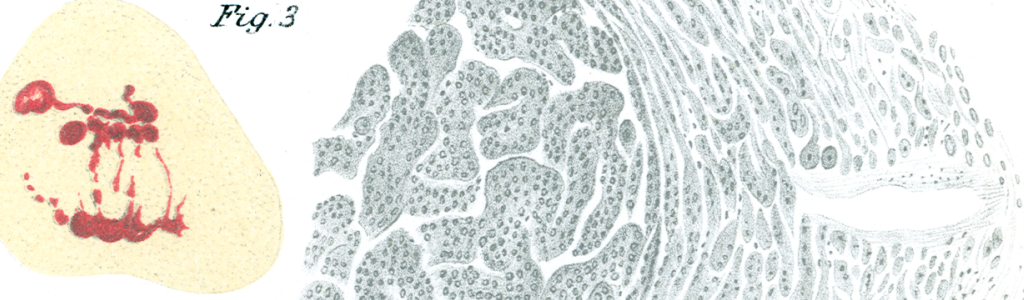

Fig. 4

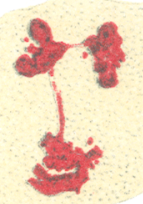

Fịg. 5
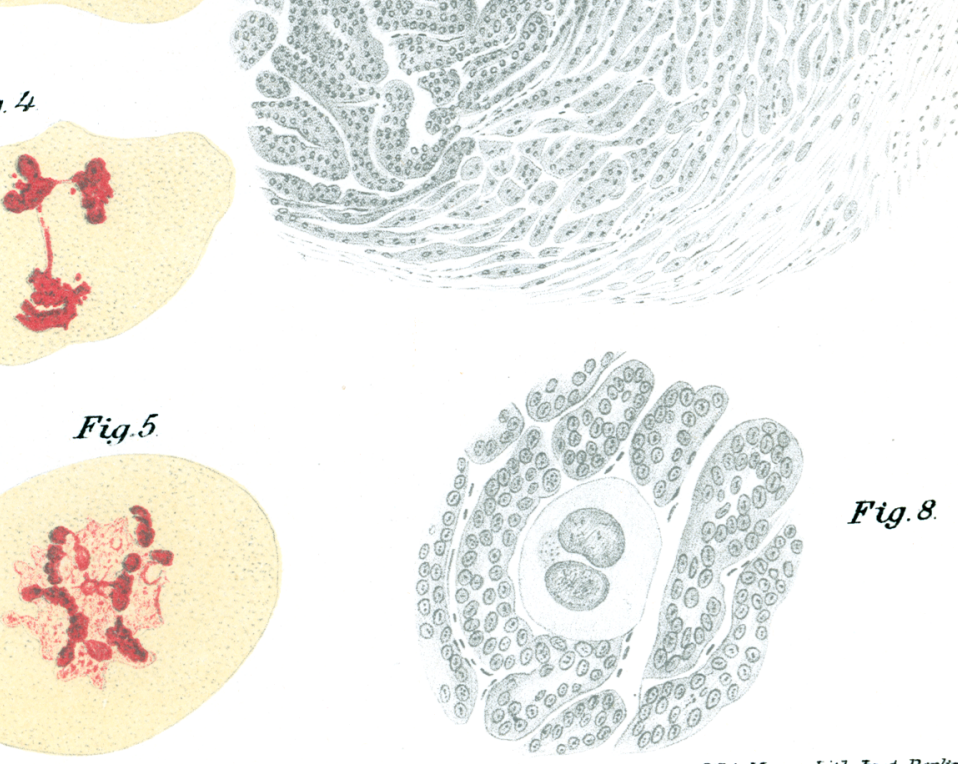
fasser unserer Lehrbücher der speciellen pathologischen Anatomie fast alle auf Einzelbeobachtungen näher eingehen, wenn sie auf die in Rede stehenden Neubildungen zu sprechen kommen. Ein allen gemeinsamer Typus ist noch nicht gefunden, ja es erscheint fraglich, ob er je gefunden werden wird, ob er überhaupt existirt! Die Ursache für diese Mannigfaltigkeit glaube ich darin in erster Linie erblicken zu dürfen, dass eine ganze Classe der Adenome sich in Lebern entwickelt, welche bereits vor der Entstehung der Neubildung verschieden hohe Grade von Veränderungen erfahren haben. Wenn man weiter nichts vor sich hätte, als die atypische Wucherung einer normal gebauten Drüse, dann wären aucb die sich ergebenden Bilder mehr oder weniger gleich, und dann auch mehr oder weniger eindeutig. Also der pathologisch veränderte Mutterboden bildet einen wesentlichen Factor bei der Histogenese der Leber-Adenome, und da ist denn eine weitere, vielleicht die allerschwierigste Frage die: stehen die älteren Veränderungen stets in Zusammenhang mit der Adenom-Bildung und in welchem? Endlich kommt hinzu, dass progressive Veränderungen und regressive so Hand in Hand laufen, dass nur eine sehr genaue Untersuchung vor Tragschlüssen bewahren kann.

Die Schwierigkeit der Beurtheilung der Leber-Adenome kam ganz besonders auf der 66. Versammlung deutscher Naturforscher und Aerzte zu Wien 1894 zum Ausdruck. Als daselbst Herr Kretz Präparate circumscripter knotiger Hyperplasie des Lebergewebes und Compensation bei Schwund des Lebergewebes demonstrirte, und als sich an seinen Vortrag eine lebhafte Debatte anschloss, an der sich ausser dem Vortragenden die Herren Eppinger, Lubarsch, Chiari, Ponfick, Markwald, Rindfleisch, Beneke, Thoma, Weichselbaum betheiligten, und ihre Ansicht durch eingehende Detailstudien begründeten, da kam die Frage keineswegs zu einem allseitig befriedigenden $A b$ schluss: Wo liegt die Grenze zwischen Hyperplasie und AdenomBildung? Das Resultat war und blieb auch hier, dass sich eine scharfe Grenze vorläufig nicht ziehen lasse. Die eigenthchen Characteristica, das circumscripte knotenförmige Auftreten, die Kapselbildung, der Druck auf das Nachbargewebe, die MetastasenBildung nach eventueller maligner Entartung, endlich die wechselnde Betheiligung der verschiedentlichen Elemente der 
Leber selbst, alles das sind Gesichtspunkte, die zur Beurtheilung herangezogen sind, die aber doch immer noch in einer Reihe von Fällon versagen und eine definitive Entscheidung nicht herbeiführen.

In demselben Jahre, als diese Naturforscher-Versammlung tagte, erschien eine Arbeit von Frohmann, die uns gleichzeitig mit der exacten Beschreibung eines Falles eine vollständige Literatur-Zusammenstellung giebt, und dadurch einen Ueberblick ïber die einschlägigen Veröffentlichungen bedeutend erleichtert. Die wichtigsten Arbeiten unserer älteren Literatur über Leberadenome, welche schon in der Frohmann'schen Arbeit Berücksichtigung finden, will ich nur kurz berïhren; in fast einer jeden von ihnen findet sich eine kritische Besprechung der einschlägigen Literatur.

Die wichtigsten hierher gehörigen Arbeiten stammen von Rokitansky, Wagner, Griesinger, Rindfleisch, Friedreich, Klob, Hoffmann, Eberth, Willigk, Wulff, Perls, BirchHirschfeld, Jungmann, Greenish, Pawlowski. Sie haben grösstentheils einschlägige Fälle beschrieben, in den meisten Fällen sind die multiplen Adenome mit Cirrhose combinirt; die Träger waren meist Männer und standen in mittleren Jahren, oder waren älter. Einzelne Autoren setzen schon die Geschwulstbildung mit gewissen Regenerations-Vorgängen in Parallele. Meist wird jeder Zusammenhang zwischen Cirrhose und Adenom-Bildung in Abrede gestellt, und beides für zwei selbständige, neben einander bestehende Processe gehalten. Viele haben den Uebergang von Leberzellen in Geschwulstzellen verfolgt.

Dann folgt eine wichtige Arbeit über die knotige Hyperplasie und das Adenom der Leber von Simmonds; ausgehend von einigen eigenen Beobachtungen giebt er zum ersten Male eine meiner Auffassung wach durchaus befriedigende Classification. Freilich waren hierzu auch früher schon Versuche gemacht; doch erwiesen sich die gegebenen Eintheilungen nicht als ausreichend, Es lässt sich freilich nicht leugnen, dass man wohl vereinzelte Uebergänge von einer zur anderen Form finden kann, die sich daher der gegebenen Eintheilung nicht zwanglos fügen; aber die wesentlichen Gesichtspunkte sind sicher bei der Simmonds'schen Eintheilung maassgebend gewesen, und die 
Uebergangsformen, sowie Combinationen mehrerer Furmen in derselben Leber machen natürlich eine an sich richtige Eintheilung nicht werthlos.

Wie gesagt zieht er die knotigen Hyperplasien der Leber ebenfalls mit in Betracht, und er ist der erste, der scharf unterscheidet zwischen solitären knotigen Hyperplasien, die in ganz gesunden Lebern vorkommen, und die er für angeborene Missbildungen hält, wie auch schon Klob aussprach, und zwischen multiplen knotigen Hyperplasien, welche gewissermaassen eine Ersatzwucherung darstellen bei degenerativen Veränderungen des Leber-Parenchyms. Seine Darstellung, die er ausserdem durch viele Beispiele aus der Literatur stïtzt, ist nicht allein völlig iberzeugend, sondern man kann sie leicht an entsprechenden Präparaten jederzeit nachprüfen. Ich selbst bin in der Lage gewesen, häufiger beide Arten von Leber-Hypertrophie, die erste in gesunden Lebern, die zweite in Lebern zu finden, wo LeberEpithelien zu Grunde gegangen waren. Ferner erinnere ich nur an die sogenannte grobknotige Leber-Cirrhose, oder an den besonders auch von Marchand beschriebenen Ausgang der acuten Leber-Atrophie in multiple knotige Hyperplasie. Was die solitären knotigen Eyperplasien, deren Charakter natürlich dadurch nicht geändert wird, dass auch sie gelegentlich zu zweien oder dreien in derselben Leber gesehen werden können, anbetrifft, so stimme ich auch hierin ganz mit Klob und Simmonds überein, und halte sie ebenfalls für Störungen aus der Zeit der Leberentwickelnng. Da ich jedoch in einer gesonderten Arbeit hierauf einzugehen gedenke, so unterlasse ich es an dieser Stelle.

Die zweite Hälfte seiner Untersuchangen betrifft das eigentliche Adenom: Indem er zunächst die multiplen Adenome bespricht, beschreibt er einen Fall, der uns für diese Kategorie als Typus gelten kann, einen Fall von Cirrhose bei einem 75jährigen Säufer mit multiplen Adenomen. Dieser Fall stimmt in vieler Beziehung mit dem von mir später beschriebenen überein. Er stellt dann fest, dass in fünfzebn in der Literatur bekannten identischen Fällen vierzehn Mal Cirrhose gleichzeitig vorhanden war; auch er hält die Cirrhose für den älteren Vorgang, an den sich secudür Adenom-Bildung angeschlossen hat. Somit entstehen die multiplen Adenome in schon erkrankten Lebern. Die vierte 
Kategorie bildet das solitäre Leber-Adenom; auch dafür giebt es Beispiele.

Auf diese Arbeit glaubte ich aus dem Grunde näher eingehen zu sollen, weil in der gegebenen Classification der Charakter der verschiedenen Bildungen mit derjenigen Schärfe gezeichnet ist, wie es überhaupt in diesem Falle möglich ist. In je einer der beiden Hauptgruppen, den beiden multiplen Veränderungen, kommen ätiologische Momente in Betracht; es liegen schon vorher schwer veränderte Organe vor; der Charakter der Neubildung ist abhängig von seinem Mutterboden, und es soll im Folgenden, wenn ich meinen Fall beschrieben habe, darauf eingegangen werden, dass es die Aetiologie ist, die bei der Classification eine massgebende Rolle spielen muss, und dass mein Fall, der sich der dritten Gruppe von Simmonds angliedert, sehr wohl geeignet ist, dessen Ansicht zu bestätigen, ja die bei ihm nur angedeuteten ätiologischen Momente näher zu präcisiren.

Weiter folgen Arbeiten von Staats, Homann, Hansemann, Beneke. Letzterer wendet sich gegen die Eintheilung von Simmonds, weil darin der Unterschied zwischen regenerativer und geschwulstartiger Neubildung zu wenig berücksichtigt sei. Im Hinblick auf meinen Fall bin ich zu der Ueberzeugung gelangt, dass man nach diesem Gesichtspunkt eine Eintheilung nicht machen sollte; denn es kommt darin regenerative Neubildung und Geschwulstbildung eng mit einander verknüpft vor, die erste geht in die zweite über; die beiden Fälle, die er dann schildert, fügen sich, meine ich, der vierten Simmonds'schen Gruppe an.

Orth bespricht die Leber-Adenome sehr ausführlich mit Berücksichtigung einzelner Fälle. $\mathrm{Er}$ hebt die makroskopische Aehnlichkeit der multiplen Leber-Adenome mit multipler knotiger Hyperplasie, wie sie z. B. bei der grossknotigen Leber-Cirrhose beobachtet wird, hervor. Im Verlaufe seiner weiteren Betrachtungen sagt er, indem er von multiplen Leber-Adenomen in Combination mit Cirrhose spricht: "Man wird unwillkürlich zu der Frage angeregt, ob nicht etwa hier eine, aus irgend einer, leider unbekannten Ursache entstandene vicariirende Hypertrophie über das Ziel hinansgehe und durch atypisches Wachsthum zu Geschwulstbildung führe." Im Anschluss hieran wird der Ueber- 
gang in Carcinom besprochen. Es ist hier zum erstenmale deutlich eine Ansicht zum Ausdruck gebracht, welche, in der Voraussetzung, dass die Cirrhose das ältere Leiden sei, diese Cirrhose in eine ganz bestimmte Beziehung zur Adenombildung bringt, einen Causalconnex zwischen beiden herstellt. Ich bin, wie meine weiteren Erörterungen zeigen werden, auf Grund eigener Beobachtungen von der Richtigkeit dieser Annahme fest überzeugt und bin verwundert, in Arbeiten, wie die von Frohmann und von Siegenbek van Heukelom, die ich sogleich besprechen will, nur ganz kurze Andeutungen über den interessanten Zusammenhang zwischen Cirrhose und multipler AdenomBildung zu finden. Bei Frohmann (S. 23) lese ich: "Was schliesslich die Beziehungen der Leber-Cirrhose zur Entstehung der multiplen malignen Adenome betrifft, so muss ich gestehen, dass die Auffassung von Sabourin, Simmonds, Orth u. a.m., rein theoretisch betrachtet, viel Verlockendes für sich hat. Diese Autoren stellen sich den Zusammenhang derart vor, dass die bei der Cirrhose als Compensations-Vorgang aufzufassende Zellhypertrophie in Folge eines unbekannten Reizes über ihr Ziel hinausschiesse und dann zar Geschwulstbildung führe; auch ich war anfangs von der Richtigkeit dieser Hypothese überzengt..." Zum Schluss sagt er: "Ich schliesse mich daher denjenigen Autoren an, welche beide Processe als unabhängig vọn einander betrachten." - Grade ein Untersucher, wie Frohmann, welcher die Literatur völlig durchgearbeitet und weiterhin die Vorgänge an den Leberzellen bei der Adenom-Bildung genau studirt hat, sollte die immer and immer in so typischer Weise wiederkehrende Combination von Lebercirrhose und multipler Adenombildung für ein Spiel des Zufalles halten? Auf die Begründung seiner Ansicht komme ich weiter unten noch zurück.

Frohmann beschreibt selbst einen Fall von schwerer Cirrhose und multipler Adenom-Bildung bei einem 37-jährigen Schiffer. Es wird der hier offenbar ungewöhnlich hohe Grad von Gallengangs-Neubildung besprochen. Im Epithel der gewucherten Gallengänge finden sich Mitosen; er schildert weiter hypertrophische Vorgänge an den Leberzellen, dann geht er auf die Geschwulstbildung selbst ein und beschreibt, wie überall die Tumorzellen aus Leberzellen hervorgehen und sich dann unter sich, unter 
Anftreten zahl'eicher Mitosen, vermehren. In den einzelnen Tumoren finden sich häufig Riesenzellen, die zahllose Kerne in sich bergen. Diese Riesenzellen sind seiner Meinung nach durch Confluenz mehrerer Geschwulstzellen entstanden. Die Tumoren sind an vielen Stellen in Blut- und Lymphgefässe eingebrochen; in den Lungen finden sieh gleich gebaute Metastasen. Hierauf bespricht er die einschlägige Literatur und schliesst mit einer Betrachtung über amitotische Theilung an Leberzellen.

Seit dieser Arbeit und der Streifung unseres Gebietes auf der Versammlung deutscher Naturforscher und Aerzte in Wien 1894, die ich oben schon erwähnt habe, sind meines Wissens nur noch zwei eingehende Bearbeitungen über das Leberadenom erschienen, auf die ich näher eingehen muss, zumal da sie in der Frohmann'schen Arbeit noch nicht berücksichtigt werden konnten.

Siegenbeek van Heukelom hat drei Fälle von LeberAdenom beschrieben, welche wiederum alle mit Cirrhose combinirt waren. Ebenso wie Simmonds fasst er die Multiplicität der Geschwülste als das Haupt-Characteristicum auf; er giebt eine Tabelle über die bisher beschriebenen Leber-Adenome, mit genauer Angabe von Geschlecht und Alter des Patienten, Dauer der Krankheit, Gewicht der Leber, Art der Tumoren in makroskopischer und mikroskopischer Beziehung, sowie über das gleichzeitige Vorhandensein von Cirrhose, Ascites, Icterus, Vermehrung der Gallengänge, Venenthrombose und Metastasen in anderen Organen. Indem er seine Fälle vor Augen führt, trennt er streng die Cirrhose von der Neubildung; letztere beginnt mit kleinen Anfängen; ein kleiner Bezirk bietet Veränderungen der Zellen, noch sind es Leberzellen, aber vergrösserte und in ihrer Lage zu einander veränderte; noch sind sie von endotheltragenden Capillaren umgeben und noch führen sie Gallengänge zwischen sich; weiterhin gehen sie in Tumorzellen über. Er beschreibt ferner, wie in seinem ersten Falle Geschwulstmassen in den Pfortaderästen liegen, hat aber das Hineinwachsen nicht deutlich sehen können. Es befanden sich in dieser Leber einige grössere Geschwulstknoten, und er schliesst die Möglichkeit aus, dass hierin die primären Tumoren zu erblicken seien, und alle anderen alsdann a.ls Metastasen aufzufassen seien. Die Zellen des Tumors 
sind in diesem Falle grösser als Leberzellen, und Riesenzellen mit einzelnen grossen oder mehreren kleinen Kernen sind zwischen ihnen anzutreffen. Da, wo die Geschwulstbildung beginnt, findet sich ausserdem grosse Polymorphie der Kerne sowohl, wie der Zellen. Er beschreibt in diesen Zellen amitotische Zerlegung des Riesenkèrnes in viele kleine Kerne, zu deren Darstellung er eine besondere Färbung angiebt.

Sein zweiter Fall ist ein ähnlicher; besonders deutlich finden sich Stellen, wo Leberzellbalken durch Zellwucherung und Bildung eines Lumens den Charakter der embryonalen Leber annehmen; er schildert ferner die Entstehung von Leberzellsäulen im cirrhotischen Bindegewebe, welche er als regressive Veränderungen auffasst, nicht als Neubildung. In diesem zweiten Falle stammen die Tumorzellen auch wieder deutlich von den alten Leberzellen $a b$, sind aber beträchtlich kleiner.

In seinem dritten Falle fanden sich wieder zahllose hypertrophische Leberzellen mit grosser Polymorphie ihres Leibes und ihrer Kerne; wiederum gehen die Tumorzellen aus Leberepithelien hervor, die junge Brut ist eher kleiner als Leberzellen, aber unregelmässig.

In einer Epikrise über seine Fälle kommt er zu dem Schluss, dass die Neubildung mit einer Veränderung der Leberzellkerne beginnt, die dann secundär das Protoplasma in Mitleidenschaft zieht, und zu atypischer Wucherung anregt. Erst in einem Schlusswort streift er die Frage eines Zusammenhanges zwischen Cirrhose und Adenom-Bildung; er sagt, es müsse ein Zusammenhang bestehen, den er, ebenso wie andere Forscher, nicht habe finden können. Er nimmt ebenfalls an, die Cirrhose sei älter; dass sie jedoch die Ursache sein könne, hält er für ganz unbewiesen.

Endlich hat Marckwald eine Anzahl von Fällen beschrieben und beurtheilt. Da ich die Präparate nicht kenne, kann ich nicht beurtheilen, wie weit seine Fälle hierher gehören. Er hält die Cirrhose für eine Folge-Erscheinung der Adenom-Bildung. Diese und noch andere seiner Folgerungen sind für mich ungleich weniger überzeugend; ich finde in meinem sogleich zu schildernden Falle keinerlei Anhaltspunkte für seine Auffassung. 
Durch die Güte des Herrn Dr. L. in Bremen ging nun dem pathologischen Institut in Göttingen ein Fall za, den ich im Folgenden zu beschreiben gedenke. Er bietet im Einzelnen ausserordentlich viel Interessantes, und ist auch weiterhin, als Ganzes betrachtet, sehr geeignet, die Auffassung über die AdenomBildung in cirrhotischen Lebern zu fördern; so sei mir denn eine möglichst genaue Beschreibung dieser Leber gestattet. Der Uebersendung der bei der Section gewonnenen Leber folgte folgender Bericht des Herrn Dr. L.:

„Patient starb, gerade 48 Jabre alt, am 26. Mai 1899 Nachmittags; Obduetion am 27. Mai Morgens. Er war Kaufmann; ich kannte denselben ärtlich seit 1883. Er lebte seit 1873 in Südamerika, seit 1889 meistens in Bremen, war jedoch bäufig wieder in Südamerika. Die Frage nach Lues ist verneint worden; Fran und sechs Kinder im Alter von 9-18 Jahren zeigen keine Symptome dieser Krankheit. Er ist wohl als „Potator" zu bezeichnen. Er ging im Herbst 1898 wieder nach Südamerika, kehrte am 1. Mai bierber zurück und wurde von mir in das St. Josephs-Spital aufgenommen. Schon im Jahre 1893 war zuerst hochgradiger Zuckergebalt des Urins constatirt worden, welcher jedoch bei passender Diät und nach einer Cur in Karlsbad gänzlich und für längere Zeit verschwand, aber sich in späteren Jahren doch immer wieder geltend machte. Am 1. Mai 1899 waren $6 \mathrm{pCt}$. vorbanden, welche jedoch im St. Josephs-Stift vollständig wieder verschwanden. Seit 1893 , oder schon früber, traten verschiedentlich leichte Anfälle von Arthritis urica auf. Seit mindestens zwei Jahren besteht Verdacht auf Lebercirrbose wegen grosser Härte der Leber und ieterischer, schmutziger Hautfarbe. Bei seiner Aufnahme in's Josephs-Stift erforderte hochgradiger Ascites die Punctio abdominis, welche klare Flüssigkeit ergab; es entwickelte sich rasch allgemeiner Hydrops, und es erfolgte der Tod an Erschöpfung, nachdem Tags zuvor sehr copiöse, blutige (früher nicht beobachtet) Stublentleerungen eingetreten waren. Da der Fall mir klinisch absolut klar zu liegen schien, wurde leider nur die Eröffnung der Bauchhöhle gemacht. Seitens der Lunge oder anderer Organe waren keinerlei krankhafte Symptome vorhanden gewesen."

Da uns nur die Leber übersandt wurde, giebt der Absender über den übrigen Leichenbefund noch folgende Angaben: „Im Baucbe reichliche Jengen eitrig getrübter Flüssigkeit; Magen und Darm mit Blut gefülllt, die Venen um die Cardia stark ectatiseh; Magenschleimhaut zeigt nur catarrhalische Veränderungen. Milz schlaff, das $1 \frac{1}{2}-$ bis 2 fache der normalen Grösse betragend, Nieren zeigten makroskopisch keine Veränderungen; Weiteres kann ich leider nicht berichten."

Hierauf sandte das Pathologische Institut zu Göttingen folgenden, vorJäufigen Bericht: 
"Die zahlreichen gelblichen, über die Schnittfäche vorspringenden Knoten in der cirrhotiscben Leber erweisen sich mikroskopisch als adenomatös gebaute Partien. Die Zellformen, welche die Adenom-Stränge enthalten, sind sehr wechselnde, in der Bauptmenge kleine, polyedrische, dazwischen grosse, polymorphe, mit knospenden Kernen versehene Zellen, vereinzelt auch Riesenzellen mit Riesenkernen. Es wird eine genaue Untersuchung dieses Falles im Institut stattfinden und später Bericht erstattet werden."

Bei der makroskopischen Betrachtung unserer Leber liegt ein in allen Dimensionen leicht vergrössertes Organ vor, das in seiner äusseren Gestalt den Typus einer grossknotigen Lebercirrhose darbietet. Das auf dew Untersuchungstisch liegende frische Organ passt sich nicht schlaff seiner Unterlage an, sondern behält seine Form fast völlig, auch bei Lageveränderungen, bei. Der tastende Finger bestätigt die allgemeine Induration der Leber; geschwunden ist die Brüchigkeit, die relativ leichte Zerreisslichkeit und weiche Consistenz des normalen Organs. Die Vergrösserung ist, wie gesagt, im Ganzen nur eine mässige; nichtsdestoweniger sind die normaler Weise scharfen Ränder sebr erbeblich abgestumptt, und die ganze Gesammtform ist etwas klumpiger, plumper geworden, erinnernd an die Conturen, die sonst nur sehr stark vergrösserte Lebern darbieten. Ich weiss nicht, ob man bei dieser Schilderung errăth, was ich bezeichnen will: Man glaubt dem Organ anzusehen, dass der Vergrösserung eine Schrumpfung in toto vorhergegangen ist, die dann einer Grōssenzunahme gewichen ist, welche auf Kosten der weiter unten zu beschreibenden knotigen Neubildungen zu setzen ist. Der ganze linke Leberlappen ist im Verhältniss zum rechten recht klein; ich bin jedoch der Meinung, dass es sich hier um eine angeborene Anomalie handelt; denn die weiterbin zu schildernden Veränderungen, sowohl progressiver, wie regressiver Natur, sind in beiden Hälften etwa gleich weit fortgeschritten. In dieser Beziehung ist deshalb dieser Fall nicht geeignet, die Beobachtung von Siegenbeek van Heukelom zu bestätigen, der den rechten Lappen stets mebr ergriffen fand, als den linken. Die Oberfäche ist äusserst grobhöckerig; während die durchschnittliche Grösse dieser Höcker etwa einer balbierten Erbse entspricbt, kommen dazwischen eingestreut zahllose k]einere Granula zum Vorschein, und grössere, bis $1 \frac{1}{2} \mathrm{~cm}$ im Durchmesser messende Buckel. An der Unterfläche der Leber überwiegen im Allgemeinen die grösseren Formen, besonders im Gebiet des Lobulus Spigelii, weleher durch grosse, zum Theil confluirende Knoten in eine knollige Hervorragung verwandelt ist. Der vordere, scharfe Rand ist, wie schon gesagt, abgestumpft; er bildet, entsprechend dem höckrigen Bau, nicht eine gleichmässige, stumpfe Kante, sondern ein äusserst unregelmässiges Profil. Die Leberkapsel zeigt eine gleichmāssige Verdickung, und liegt wie ein dichter Schleier über den Lebergewebe. Doch auch dureh die verdickte Kapsel hindurch schimmern verschiedene Farben, und unschwer erkennt man, dass durchängig die tieferen Furchen grauröthliches Gewebe, die kleineren 
Granula theils rostbraunes, theils auch dunkelgrünes, die grösseren Buckel endlich ein hellgelbliches Gewebe in ihrer Tiefe bergen. Auf der Höhe der grösseren Höcker ist die Kapsel straffer, und erscheint dünner, als in den dazwischen verlaufenden Furchen. Von den Ansatzstellen der Leber-Ligamente aus zieben strahlige, beträchtliche Verdickungen in die Kapsel binein und tragen dazu bei, das Oberflächenbild zu einem äusserst bunten zu machen. Die Kapsel ist überall glatt und spiegelnd, und zeigt nirgends Verwachsungen mit der Umgebung.

Eine genaue Untersuchung des Leberhilus ergiebt keinerlei patbologische Veränderungen: Die Gefässe sind leer, insbesondere frei von Gescbwulstbildung, die Gallenblase ist von normaler Gestalt und Grösse; ibre dunkelgrün gefärbte Schleimhaut ist völlig unverändert, ebenso auch die grossen Gallenwege. Eine Untersuchung der Hilus-Lymphknoten ergiebt ebenfalls keinen pathologiscben Befund, und ich will gleich bier vorwegnehmen, dass auch mikroskopisch keine wesentliche Veränderung zu entdecken war. Nachdem wir nur noch constatirt haben, dass das ganze Organ für seine Grösse recht schwer ist, und dass bei der Betastung die grösseren Höcker sich weicher anfüblen, als die kleinen, insbesondere aber als das graue $Z$ wischengewebe, gehen wir zur Betrachtung von Durchschnitten über.

Während das Messer durch die Lebersubstanz gleitet, knirscht es stellenweise, und man hat die deutliche Empfindung, dass man ein Gewebe von sebr wechselnder Beschaffenheit durchschneidel. Sobald das Messer das Gewebe getrennt hat, stellt sich eine äusserst höckerige Scbnittfläche her, welche der Leberoberflâche vergleichbar ist und durch schnelles Hervorquellen der massenbaften, grösseren und kleineren, gelbweissen Tumoren entsteht, die schon durch die Kapsel von aussen erkennbar waren; je grösser der Knoten ist, um so mebr quillt er auf der Schnittfäche bervor, ja der Gewebsdruck der grösseren ist so beträchtlich, dass die hervorquuellenden Tumoren da, wo sie dicht stehen, die zurückbleibenden Abschnitte förmlich überlagern und so dem Blicke entziehen. Das Hervorquellen der Tumormasse wird ermöglicht durch ibre ausserordentlich weiche Consistenz, welche gestattet, dass man ganze Gewebsbröckel mit leichtem Wasserstrahl abspült. Lieser Zerfall, vereinigt mit hellerer Färbung des Centrums, lässt schon makroskopisch eine centrale Nekrose bei den grösseren Kanoten vermuthon. An diesen frischen Schnitten durch das Präparat sei nur noch erwäbnt, dass der Blutgehalt ganz ausserordentlich herahgesetzt ist. Auch die grösseren Gefässe enthalten durchweg nur. Spuren von flüssigem Blut oder postmortalen Gerinneeln. Leider ist mir über den Blutgehalt des Organs bei der Herausnahme nichts bekannt.

Wegen der geschilderten Beschaffenheit der frischen Durcbscbnitte halte ich es für instructiver, eine genaue makroskopische Untersuchung an gehärteten Stücken vorzunebmen. Ich beschreibe daber nunmebr die nach der Härtung völlig glatte Schnittfläche. Diese Härtung wurde in Formol-Müller 
vorgenommen: sie hat das bunte Aussehen der Schnittfäche nur wenig abgeschwächt.

Das ganze Organ ist von starker Bindegewebs-Wucherung durchzogen; es liegt eine schwere annuläre Cirrbose vor. Von gleicbmässiger Stärke in allen Theilen, ziehen die faserigen, narbenartigen, grauen Stränge, breit ansetzend an der Kapsel und an den Gefässen, durch das Parenchym und ringförmig herum um zahllose Inseln von verschiedener Grösse, Färbung und Gestalt. Weite Blutgefässe klaffen darin, meist kreisrund, jedoch in der Nähe grösserer Geschwulstknoten häufig von zwei oder mehreren Seiten comprimirt, oder es springen buckelige Hervorwölbungen in ihr Lumen hinein, über welche überall die intacte Intima hinwegzieht. In der Nähe der Gefässe oder auch in der Umgebung grösserer Geschwulstknoten, die in ibrem Wachsthum das umschnürende cirrhotische Bindegewebe nach allen Seiten $\mathrm{zu}$ verdrängen streben, führt es theils dunkelbraunrothes, theils gelbliches Pigment. Ueberall hat man den Eindruck eines änsserst chronisch verlaufenden indurativen Processes; mit wenigen Ausnabmen jst eine scharfe Grenze zwischen dem Bindegewebe und den eingelagerten Inseln vorbanden.

Unter diesen Inseln kann man auf den ersten Blick zwei verschiedene Arten unterscheiden: Inseln von erhaltenem Lebergewebe, und Inseln, welche aus Neubildung bestehen. Ueberall sind, allein schon durch die Farbe, beide Arten auf's Deutlichste von einander zu unterscheiden.

Die ersteren, die Inseln von erbaltenem Lebergewebe, sind kleiner, überwiegen jedocb an Zahl die andern; auf frischen Schnittflächen springen sie viel weniger bervor, sie sind von unregelmässiger Gestalt und tragen die Spuren des Druckes, welcher von dem cirrhotischen Bindegewebe einerseits, von ihren jungen Schwesterbildungen andererseits ausgeht, deutlich zur Schau; ihre Farbe ist ein mehr oder weniger intensives Braunroth bis Rostfarbe, wieder an anderen Stellen haben einige, meist benachbarte Leberinseln die Farbe des grünen Icterus.

Die zweite Art der Inseln ist ganz homogen gelblichweiss gefärbt, und charakterisirte sich schon auf der frischen Schnittfläche durch das mächtige Hervorquellen. Aber auch die gehärteten Tumoren bieten alle Merkmale schnell wachsender Geschwülste dar, die einen heftigen Druck auf die Nachbarschaft ausüben; ihre helle Farbe dentet auf eine rein zellige Nenbildung hin; der Durchmesser dieser Knoten schwankt zwischen den kleinsten, mit blossem Auge gerade noch sichtbaren, bis zu etwa $1 \mathrm{~cm}$ im Durchmesser baltenden Knoten, die dann häufig fast confuiren, sich gegen einander abplatten, aber bei genauem Zusehen doch stets noch durch einen bindegewebigen Strang von einander getrennt sind. Die grösseren Knoten tragen in ihrer Mitte eine oder mehrere Erweichungs-Höhlen. Schon makroskopisch kann man ein Drtheil gewinnen, wovon diese Geschwülste ausgehen: Die kleinsten von ihnen liegen nehmlich ausnahmslos theils central; theils excentrisch in den braunen Lebergewebs-Inseln, die vorher beschrieben sind; indem sie sich vergrössern, drängen sie ihren Nutterboden immer mehr und mehr auseinander, so dass 
dieser sie in vielen Fällen nur noch wie eine zarte, braune Kapsel umbüllt, die dann wiederum von cirrhotischem Bindegewebe unkapselt ist. Endlich verschwindet auch dieser Rest, und die Neubildung hat völlig die LebergewebsInsel substituirt. Ausserbalb von diesen Lebergewebs-Inseln, also im cirrhotischen Bindegewebe, sieht wan keine selbständigen jungen Tumoren aufspriessen, aber von grösster Wichtigkeit ist es, dass manche dieser Knoten neben sich feinste Streifcben zeigen, die, sichelförmig um einen Theil ibrer Circumferenz herumgelagert, im Bindegewehe verlaufen, dieselbe Farbe wie die Tumoren haben, und auf ein Ueberschreiten der natürlich gegebenen Grenze hindeuten. Diese Verhältnisse sind jedoch so blein, dass sie sich dem unbewaffneten Auge fast entziehen, und ich verschiebe ihre Beurtheilung daber auf die mikroskopische Beschreibung. Erwähnen will ich hier nur noch, dass in ein und derselben Leberinsel bäufig nicht nur eine solche kleine Neubildung aufspriesst, sondern es können mehrere in unregelmässigen Abständen darin beobachtet werden. Zienlich gleichmässig über die ganze cirrhotisehe Leber sind diese Neubildungen vertheilt, und auch bei sorgfältiger Untersuchung findet sich keine grössere Geschwulst, die als ein Haupt- oder Primärtumor aufgefasst werden könnte. An einzelnen Stellen, namentlich von der Unterfläche des Organs, nebmen diese Tumoren freilich streckenweise fast das ganze Lebergewebe ein; dort sind dann alle Leberparenchym-Reste zu Grunde gegangen, und die zwischen ihnen verlaufenden Bindegewebs-Streifen baben unter dem heftigen Wachsthumsdruck ibren Raum erbeblich einschränken müssen.

Noch eine Besonderheit sei kurz erwähnt, die erst bei der mikroskopischen Untersucbung eingehende Berücksichtigung finden soll: Manche von den jungen Neubildungen, und zwar niebt die grössten, sondern die mittelgrossen, etwa banfkorngrossen, haben eine etwas unregelmässige, dnnkelrothe, wie hämorrbagisch infiltrirte Randzone, die nach aussen zu immer intensiver wird, und sich nach dem Centrum hin allmählich verliert.

Es eräbrigt nur noch, einer grossen Gallengangs-Cyste Erwähnung zu thun, welche, etwa kirschgross, sich mitten im rechten Lappen, scharf in das cirrbotische Gewebe eingesetzt vorfindet, und von einer dunkelbraungelben, bröckligen Inbaltsmasse erfüllt ist, welche auf Schnitten leicht ausfällt, und bei frischer Untersuchung viel Pigment verschiedener Art, in allerhand Zerfallsmassen eingelagert, enthält.

Von diesem Präparate wurden zur mikroskopischen Untersuchung viele Stücke in folgenden Härtangs-Flüssigkeiten vorbereitet:

1. Formol Müller (nach Orth): 10 Theile $40 \mathrm{pCt}$.' Wormol auf 90 Theile Müller'sehe Flüssigkeit 24 Stunden im Brütofen von Körpertemperatur. (Um diese Stücke besonders auch für das Gefrier-Mikrotom schnitffertig bereit zu halten, empfiehlt es sich, sie nach gründlicher 
A uswässerung einfach in einer ganz dünnen Formol-Lösung in Wasser, etwa $1: 200$, liegen $z$ lassen, worin sie sich beliebig lange unverändert erhalten, obne ihre Fărbbarkeit einzubüssen.)

2. Mäller'sche Flüssigkeit.

3. Alkohol von steigender Concentration.

4. Alkohol absolutus.

5. Flemming'sche Mischung.

6. Zenker'sche Mischung.

Unsere Leber kam freilich nicht mehr in völlig frischem Zustande in unsere Hände; trotzdem gelangen alle Färbungen nocb sebr zur Zufriedenheit, besonders aber hatte die Färbung der Tumorzellen in keiner Weise gelitten. Diese waren aucb der Safranin-Färbung zur Darstellung der feinsten Kernstructur noch in vollem Maasse zugänglicb.

Die mikroskopischen Schnitte wurden theils mit dem Gefrier-Mikrotom, theils nach Einbettung in Paraffin angefertigt; es kamen folgende Färbemittel zur Anwendung:

Hämatoxylin-Eosin, Lithinmearmin-Pikrinsäure, Alauncarmin, v. Giesonsche Fãrbung, Weigert's Färbung auf elastische Fasern, Safranin, Gramsche Methode.

Stellenweise waren in der Leber vorhandene Mikroorganismen wohl postmortal nicht unerheblich gewachsen; auf vielen Schnitten begegnet man in Gefässen, aber auch mitten in den Inseln von erbaltenem Lebergewebe, runden Baufen von meist stäbchenförmigen Mikro-Organismen, die natürlich für die Auffassung des Ganzen ohne Bedentung sind.

Die schwere Leber-Cirrhose, als die ganz diffuse Veränderung, stebe zunächst im Vordergrunde unserer weiteren Betrachtung. Sie trägt einen annulären Charakter, wie schon die makroskopische Betrachtung zeigte, und fast überall ist eine scharfe Grenze vorbanden zwischen den LeberparenchymResten und dem cirrhotischen Bindegewebe. Letzteres hat sich in seiner Wueherung keineswegs an die Grenzen der Leberiäppchen gehalten, sondern beliebige Trennungen mitten durch die Läppchen vorgenommen. Es trägt den ausgesprochenen Cbarakter des alten Bindegewebes, es ist faserig und kernarm und zeigt die Ersebeinungen der narbenartigen Schrumpfung. In ihm verlaufen zahlreiche Gefässe, die stellenweise durch ibre Menge und Weite den Eindruck eines cavernösen Gewebes hervorrufen. Die v. Giesonsche Färbemethode färbt dies Bindegewebe diffus roth, und nur wenig längliche Kerne, die grösstentheils äusserst schlank geworden sind, und offenbar im Verschwinden begriffen sind, liegen mit ibrer Längsachse parallel dem Faserverlauf dieses alten Bindegewebes. Die Färbung auf elastische Fasern nach Weigert zeigt uns, dass darin unendlich viele eng verflochtene und viel verästelte elastische Fasern verlaufen, die seinen āusserst zähen Charakter erklären belfen, sowie den erheblichen Widerstand, den es den wachsenden Neubildungen entgegenstellt, und der in ihrem Hervorquellen auf der frischen Schnittfläche zum Ausdruck kommt. Es sei gleich hier 
hervorgehoben, dass diese elastischen Faserzüge sich continuirlich in diejenigen Bindegewebsäste fortsetzen, welche in die Neubildungen bineinziehen. Die ursprünglichen Züge periportalen Bindegewebes sind nur noch an dem Verlanfe der Arterien, Nerven und grossen Gallengänge wieder aufzufinden. Es fehlt nun nieht völlig an frischeren cirrhotischen Partien, deren rundzellige Wucherung von den älteren Partien aus, in Form spitzzulaufender $Z$ üge in die Leberinseln vordringt, und diese in immer kleinere Theile zu zerlegen strebt. In dieser relativ jungen Brut sind, wenn auch vereinzelt und nicht immer deutlich, Mitosen zu entdecken, wenn Härtung in Flemming' schem Gemisch und Färbung in Safranin vorgenommen wurde. Auch vereinzelte gelapptkernige Leukocyten sind dazwischen anzutreffen. Fein vertheilt im Bindegewebe ist spärliches, bräunliches oder gelbliches Pigment gelegentlich vorbanden, aber bäufig ist das den jungen Geschwälsten direct anliegende Bindegewebe in einem glejchmässigen Ringe sebr dicht mit dunkelbraunem Pigment erfüllt, und man sieht, wenn man verschieden grosse Tumoren neben einander zu Rathe zieht, deutlich, wie diese pigmentirte Kapsel entsteht: es ist der Rest des nach allen Seiten an die Wand gedrückten Lebergewebes, das unter Pigment-Atrophie geschwunden ist, und an dessen Stelle die junge Geschwulst getreten ist. Nachdem die alten Leberzellen geschwunden sind, bleibt nur noch ihr Pigment zurück, das nun zwischen den länger standhaitenden Capillar-Endothelien ruht. Das Ganze lässt sich im Bau kaum von dem alten Bindegewebe trennen, nur elastische Fasern sind noch nicht darin zu entdecken.

Für die Beurtheilung unseres ganzen Falles ist es nun von äusserster Wichtigkeit, die Gallengänge genau zu untersuchen, die aucb hier massenhaft im cirrhotischen Bindegewebe zu entdecken sind. So genau ich auch meine sämmtlichen Scbnitte untersucht habe, nirgends habe ich Zusammenhänge zwischen diesen Gallengängen und den später zu beschreibenden Adenomen entdecken können. In ibnen sind fast niemals Mitosen zu entdecken, ein Lumen ist häufig nicht erkennbar, manchmal, wenn auch sehr selten, scheint ihr Epithel mehrschichtig zu sein, in den meisten Fällen aber ist es sogar sehr flach. Nirgends sah ich in diesem Fall Bilder; wie sie z. B. Frohmann beschrieben und abgebildet bat, von massenbafter Knospenbildung an den interacinösen Gallengängen; und noch eine Beobachtung ist es hanptsächlich, die mich zu der Ueberzeugung bringt, dass auch dieser Fall nur für die Auffassung zu verwertben ist, dass die interacinösen Gallengänge bei Cirrhose zum grossen Theil nicht neugebildet sind, sondern Ueberreste des zu Grunde gegangenen Parenchyms. Es sind nebmlich stellenweise rundliche Bezirke vorhanden, die nach Grösse, Lage und Gestalt zweifellos das Gebiet einer ehemaligen Leberinsel darstellen, welche von allseitig eindringendem, cirrbotischem Bindegewebe ganz aufge]öst und zerstört ist; $z$ wischen diesem relativ jungen Bindegewebe liegen dann unregelmässig geschlängelte Zellenreihen, die gar keine WachsthumsErscheinungen darbieten und mit den interinsulären Gallengängen die grösste 
Aehnlichkeit baben; sie lassen sich kaum anders auffassen, wie als Reste des zu Grunde gerichteten Parenchyms. In seinem Lehrbuche (1887, S. 940) schildert Ortb dieselben Beobachtungen bei der gewöbnlicben Cirrbose und deutet sie in diesem Sinne.

Absichtlich streife ich diese Frage hier nur mit einigen Bemerkungen, die keineswegs Beweise liefern sollen; ich thue dies deswegen, weil ich die Ueberzeugung babe, dass unser Fall für Untersuchungen in dieser Richtung nicht sehr geeignet ist, steht doch diejenige Neubildung so sehr im Vordergrande, die ron den Leberzellen selbst ausgeht; und dennocb! Gerade der letzte Umstand unterstützt mich in meiner Auffassung der interacinösen Gallengänge. Sollten gerade diese sich an dem diffusen Neubildungs-Process nicht betbeiligen, der sich hier in allen Theilen des Organes geltend macht, wenn sie junge, wachsthumskräftige oder gar schon in Proliferation stehende Gebilde wären? Sie liessen sicherlich diesen allgemein einwirkenden Reiz nicht spurlos an sich vorübergeben, und Gallengangs-Adenome würden entstehen, nicht Leberzellen-Adenome! - Doch davon später.

Die Leberzell-Inseln, welche $z$ wischen dem eirrhotischen Bindegewebe erhalten sind, stellen in ibrer Gesammtmenge einen Rest von Parenchym dar, der schätzungsweise höchstens den vierten Theil von dem Lebergewebe darstellen mag, das einem erwachsenen Menschen zukommt. Sab dieser Rest schon makroskopisch äusserst comprimirt und braun atrophisch aus, so finden wir ihn erst mikroskopisch in einem noch schlechteren Zustande; ich sage nicht zu viel, wenn ich behaupte, dass man kaun ein völlig normales Leberläppchen auffinden kann, und die matte Färbung, die Kerne und Protoplasma rait allen Färbemitteln annebmen, deutet schon auf ein Gewebe von äusserst geringer Lebens-Energie hin. Besonders ist es der Safranin-Färbung fast unzugäuglich, und in den grösseren oder kleineren Zellleibern sind $z$ wischen den mannigfachen Pigwent-Ablagerungen häufig genug nur vereinzelte Chromatin-Körnchen etwas röthlich gefärbt, die die Stelle andeuten, wo der blasse Kern liegt. Sehr viel Gallen-Pigment (mikroskopisch überwiegt das dunlselgrüne) liegt im Zellleib oder dazwischen, ebenso sehr viel fein vertheiltes, braunes, altes Blut-Pigment. Die mit Osmiumsäure vorbereiteten Präparate liefern eine Schwarzfärbung des unregelmässig zerstreuten Fettes, das in grösseren und kleineren Tröpfchen die Zellleiber erfüllt. Hier will ich einschalten, dass die Safranin-Färbung bei mir keine Färbung von Gallen-Concrementen ergeben hat, wie sie von Meder beschrieben sind. Auch im Gebiete der nachher zu beschreibenden Adenome habe ich vergeblich darnach gesucht. An manchen Stellen findet sich anch frischere cirrhotische Bindegewebs-Wucherung.

Im Centrum der besser erhaltenen Inseln baben nun die Leberzellen den Versuch gemacht, durch Leistung einer regenerativen Hypertrophie das Verlorene wieder zu ersetzen. Einzelne, zu Häufchen vereinigte Zellen haben an Grösse bedeutend zugenommen, und man erkennt deutlich, dass die central gelegenen Zellen dieses Bäufchens die grössten sind. Sie sind von 
Pigment-Ablagerung fast ganz verschont geblieben, und man siebt deutlich, dass ihr Kern sich wesentlich besser färbt, dass auch ihr Protoplasma wieder Färbung angenommen bat. Ihr Bau entspricht dem einer normalen Zelle völlig, nur sind sie grősser; freilich feblt jegliches Verglejchs-Object, denn die Nachbarzellen sind auch nicht normal. Wie gesagt, nimmt diese Hypertrophie nach der Mitte zu, und dort ist es auch, wo man alsbald mehrkernige Leberzellen und Zellen mit auffallend grossen Kernen antreffen kann. Allein gleichzeitig mit der Kern-Vergrösserung und -Vermehrung treten typische Veränderungen der Chromatin-Substanz zu Tage; der Kern ist nicht gleichmăssig dunkel gefărbt, sondern das vermebre Chromatin ist theilweise zu grōsseren Conglomeraten vereinigt und zı allerhand regelmässigen Gruppen angeordnet. Es bereitet sich ganz fraglos eine progressive Veränderung vor, deren weiteren Verlauf wir nun verfolgen wollen. (Fig. I.)

Die Vermehrung der Kerre kann hierbei eine enorme werden; ich habe bis etwa 20 und mebr gezäblt; sie liegen in Mitten der Zelle auf einen Haufen vereinigt; das umbüllende Protoplasma ist eine homogene, unformliche Masse, welche alle Farbstoffe ziemlich lebbaft aufnimmt. Neben diesen Riesenzellen liegen andere von gleicher Grösse, aber nur mit einem riesenbaften Kern, der manchmal etwas am Rande eingekerbt ist, wie in beginnender Theilung begriffen. Die Kernsubstanz färbt sicb auch hier sebr intensiv und ist lebhaft gekörnt; vielfach liegen scheinbar Vacuolen darin, auch lassen sich allerhand geformte Chromatia-Klumpen und Körnehen darin wahruehmen, welche am besten durch Safranin-Färbung darzustellen sind. Jedocb diese Veränderungen der Chromatin-Substanz sind so manuigfaltig, dass es einfacher ist, an dieser Stelle auf die analogen Beschreibungen in der Arbeit von W. v. Podwyssozki jun. hinzuweisen, auf welche ich noch später genauer eingehen will. Nur noch ein Wort über die angedeutete scheinbare Vacuolen-Bildung in diesen Riesenkernen. Ich nenne sie so, weil es runde, manchmal aber auch ovale Gebilde sind, welche deutlich eine hellere Färbung darbieten, als der übrige Kernleib. Ihre scharfe, membranartige Grenze ist von fein gekörnten Chromatin-Mengen gebildet, welche an der Innenseite dieser scheinbaren Vacuole liegen; sie selbst färben sich deutlicb, allerdings etwas blass. (Fig. II.) Es handelt sich also nicht um Vacuolen; nach den zahlreichen Uebergangs-Bildern scheint bier ein Vorstadium der amitotischen Zerlegung des Riesenkernes in mehrere Tochterkerne vorzuliegen. Vereinzelt findet man anch Bilder, wie sich ein solcher Riesenkern durch offenbar ganz langsame Abschnürung eines Fortsatzes theilt, Bilder, wie sie besonders Frohmann beschrieben und abgebildet hat: der Kern dehnt sich in die Länge, nimmt dann biscuitförmige Gestalt an und geht endlich in $z$ wei Kerne äber. Es scheint mir kein $Z$ weifel zu sein, dass wir in diesen Riesenzellen Gebilde vor uns haben, welche nicht durch Zusammenfluss mehrerer Kerne und Zellen entstanden sind, sondern durch Hypertrophie einer einzigen Zelle, deren schliesslich grösser und 


\section{7}

und grösser werdernder Kern sich in verschiedenen Stadien seines Wachsthums amitotisch in mehrere zerlegt.

In allen Uebergangsstadien dieser Zellvergrösserung, die vom Rande des hypertrophisehen Bezirkes her nach der Mitte deutlich zunimmt, und dieser Kernzerspaltung haben wir ein äusserst mannigfaltiges Bild vor uns, das nun durch einen weiteren Vorgang noch complicirter wird: Es kommt in diesen hypertrophischen Leberzellen zweifellos indirecte Kerntheilung vor. Sie ist freilich wesentlich seltener als die directe, indessen giebt uns die genaue Durchforschung der Flemming-Safranin-Präparate sehr bald deutlich Karyomitosen, die man wohl Riesen-Mitosen nennen kann. In vielen solcher Riesenkerne sah ich zunächst eine eigenthümliche Anordnung des Chromatins, das zu langen, sich vielfach durchwindenden Fäden aufgeiöst ist, und die ausserordentlich intensive Färbung mit Safranin trotz gründlicher Differenzirung in angesäuertem Alkobol lässt keinen $Z$ weifel darüber, dass wir nicht ruhende Kerne vor uns haben, dass sich eine mitotische Kerntheilung vorbereitet (Fig. I). Aber damit nicht genug, auch ältere Stadien der Karyomitose bieten sich in diesen vergrösserten Leberzellen dar, (Fig. III), und häufig ist es zur Theilung der Chromatinfäden in drei Tochtersterne gekommen, zur Triasterbildung (Fig. IV).

Diese Mitosen finden sich im Wesentlichen in hypertraphischen Leberzellen von mittlerer Grösse. Sobald ibre Grösse ein gewisses Maass äberschritten hat, scheint indirecte Kerntheilung nicht mehr vorzukommen; es scheint sich die Wachsthums-Energie der Zelle in einer riesenbaften Hypertrophie völlig zu erschöpfen, und der unbekannte Reiz scheint auszúbleiben, der sie zu einem, wenn ich so sagen darf, planvolleren Wachsthum erweckt. Oder ist dieser Reix vorhanden, die Zelle kann ibm jedoch nicht mebr Folge leisten? Ich komme auf meine Auffassung von diesem verschiedenen Kerntheilungs-Typus noch zurück.

Was wird nun aus diesen Riesenzellen? Theilt sich die ProtoplasmaMasse ebenfalls, oder nicht? Tritt also auch eine Vermehrung der Leberzellen ein, oder nicht? Trotz genauen Durchforschens habe ich kein deutliches Bild von nachfolgender Zelltheilung beobachten können, bei welcher typische Leberzellen resultiren: aus diesen Riesenzellen wächst unmittelbar eine Brut hervor, die keine Leberzellen mehr sind, soudern Zellen des Tumors, den ich nun zu beschreiben gedenke, Zellen, wie sie zu ganz ungezäblten, dichten Eaufen die Bausteine der Adenome bilden, mit denen die ganze Leber dicht besät ist. Wie mag es kommen, dass dieser mannigfachen Kerntheilung keine Zerlegung der Zellen in Tocbterzellen nachfolgt, die der Mutterzelle gleichen? Sollte der vorher veränderte Zustand der Mutterzelle daran Schuld sein, oder dazu beitragen? Sollte der Organismus die feine Arbeit nicht mehr leisten können, hochdifferenzirte Zellen, Leber-Epithelien $z u$ bilden? Denn die entstehenden Tumor-Zellen sind offenbar entdifferenzirt; es sind Rücbbildungs-Vorgänge eingetreten, und njcht nur im Bau der Zellen, und damit in ibrem functionellen Werthe, sondern auch in ihrer An- 
ordnung ist eine Rückkebr zum embryonalen Typus zu constatiren; diese Aehnlichkeit mit dem embryonalen Bau der Leber wird durch eingelagerte Riesenzellen nur noch vermebrt. Oder sollte endlich die regenerative Thätigkeit der Leberzellen, die nicht bis zu Ende hat gedeiben können, jäh unterbrochen sein durch einen unbekannten Reiz, der zur Tumorbildung führte? Hier stehen wir vor der schwierigsten Fráge; hier ist die Hypertrophie zu Ende, und hier beginnt die Geschwulstbildung.

Und darnit komme ich zur Beschreibung der grossen, gelblichweissen Tumoren, welche mikroskopisch am Allerlebhaftesten in die Augen fallen, za den multiplen Adenomen selbst. Alle angewandten Färbemittel nehmen sie äusserst begierig auf; waren sie schon makroskopisch unzählig, so wächst die Zabl dieser runden Gescbwälste im Mikroskop in's Unendliche; alle Stadien liegen zur Beobachtung vor uns. Will man nun die kleinsten Anfänge untersuchen, so darf man sich nicht irre führen lassen: Die kleinen, runden Zellhaufen können eben so gut tangential abgeschnittene Segmente von der Oberfläche eines grösseren Tumors sein; die Anfänge der Geschwulst-Bildung kann man nur in denjenigen kleinen Tumoren erblicken, welche auf Scbuittreihen sich als ganz klein erweisen. An diesen nun sieht man häufig den Uebergang der immer grösser werdenden zum Theil mehrkernigen Leberzellen in Adenom-Stränge; plötzlich legt sich neben eine vergrösserte, mehrkernige Leberzelle ein Zellbaufen, der grosse, ovale, intensiv gefärbte Kerne hat, und der völlig. dem Aufbau der grossen Adenom-Knoten entspricht (Fig. VI). Diese ersten Anfänge der Tumor-Bildung finden sich an ganz beliebigen Stellen der Leberläppchen, manchmal nahe der Centralvene, manchmal in der Peripherie. In dem jungen Geschwulst-Gewebe liegen die Kerne sehr dicht bei einander, die Zellleiber sind nur klein, das Protoplasma und besonders der Zellkern färbt sicb stark, besonders giebt Hämatoxylin sebr contrastreiche Bilder. Alsbald treten nun in diesen Tumorzellen zahlreiche Mitosen auf, die kleinen Geschwülste wachsen sebr lebhaft, und bald geht die Möglichkeit völlig verloren, ihre Entstebung zu verfolgen. Denn alsbald drängen sie ibren Mutterboden zur Seite, sie wachsen aus sich heraus weiter, und $z$ war offenbar mit ganz aussergewöhnlicher Schnelligkeit. Sie drängen das umgebende Lebergewebe gewaltig zur Seite und bringen es zu vollständiger Druck-Atrophie. Den Bau dieser Tumoren kann man wohl, wie es schon Jungmann that, mit den Windungen an der Oberfäche der Grosshirn-Hemispbären vergleichen; es sind lauter Zellstränge von etwas wechselnder Dicke, mit ovalen oder manchmal etwas unregelmässigen Kernen; grösstentbeils sind die Windungen des Tumors von einer einfachen Lage platter Zellen überzogen, wie von einer Tunica propria, die sich von den Uebergangsstellen der bypertropbischen Leberzellen in Tumorstränge continuirlicb in das Capillar-Endothel verfolgen lässt. Die Windungen sind häufig verzweigt, und wenn der rein zellige Charakter dieser tubulösen Geschwülste und der heftige Druck auf die Nachbarschaft schon einen Hinweis auf ibr schnelles Wachsthum geben kann, so wird dies 
bewiesen durch die auffallend grosse Menge von Mitosen, die man in allen Stadien darin antreffen kann. Ihre völlig gleichmässige Vertheilung über die Geschwälste beweist, dass in ibnen ein gleichmässiges Wachsthum vor sich geht, und nicht nur eine Proliferation in den Randpartien. Schon Klebs unterschied an solchen Geschwülsten ein eigenes Wachsthum durch Sprossenbildung, zweitens eine excentrische Ausbreitung durch ,Infection der Nachbarschaft“. Diese Infeetion der Nachbarschaft, welche in einer adenomatösen Umwandlung der benachbarten Leberzell-Balken besteht, ist nun auch an unserem Präparat sehr wohl zu erkennen, jedoch nur bei den allerjüngsten Geschwülsten. Sobald eine sichtbare Vergrösserung des Tumors entsteht, dann gebt das angrenzende Parenchym so schnell druck-atrophisch zu Grunde, dass keine Wucherung an ihm mehr möglich ist (Fig. VII).

War der innige Zusammenhang zwischen hypertrophischen Leberabschnitten und Adenomknoten durch ihren directen Uebergang in einander anfs Deutlichste zu erkennen, so wird er noch dadurch weiter bestätigt, dass sich auch in den fertigen Tumoren manchmal vereinzelt, manchmal auch zahlreich jene Riesenzellen wiederfinden, wie sie durch Fypertrophie der Leberzellen entstanden sind. Viele von ibnen, besonders die mit einem grossen Kern, scheinen in ihrer Entwickelung still zu stehen, und unverändert im adenomatösen Gewebe liegen zu bleiben (Fig. VIII). Leider war es mir nicht möglich, was Frohmann gelungen ist, in den Adenomen auch noch normale Leberzellen vorzufinden. Die Grösse der Tumorzellen, mit Ausnabme der erwähnten Riesenzellen, ist beträcbtlich kleiner, als die normalen Leberzellen. und hierin stimmt meine Beobachtung mit den meisten andern überein; ein Lumen lässt sich in den Geschwulstwindungen nur selten nachweisen, die grosse Mehrheit sind solide Stränge. Innerhalb dieser Tumoren habe ich keine Kerntheilung auf directem Wege nachweisen können, wie sie z. B. Hoffmann beschrieben hat; der indirecte Theilungsmodus herrsebt also mindestens ganz bedeutend vor. Pigment oder Fett findet sich in den Tumorzellen nicht, in den Geschwülsten sind keine Gallengänge zu entdecken; hierauf hat auch schon Klebs hingewiesen, und gesagt, dass dieser Befund nach der hellen, niemals ikterischen Farbe aucb zu erwarten sei. Bei der makroskopischen Bescbreibung sind bereits die dunkelrothen Randpartien einzeiner mittelgrosser Geschwulstknoten beschrieben worden; mikroskopisch liegen an diesen Stellen rothe Blutkörperchen dicht gedrängt in den Zwischenräumen zwischen den Tumorsträngen. Eine Ursache hierfür habe ich nicht finden können, stelle wir aber vor, dass es sich um eine Blutansammlung in Folge benachbarten Gefässverschlusses handelt. In der Tbat findet man, wenn auch nicht nur in der Nähe solcher blutreichen Bezirke, häufig Gefässe, die durch Tumormassen von aussen comprimirt sind.

Innerbalb der Tumoren sind niemals Gefässe nachzuweisen; und so erklärt es sich, dass bäufig genug eine central beginnende Nekrose eingetreten ist; mikroskopisch ist die Kernfärbung verschwunden, und es scheint sich 
dieser Nekrose auch ein schleuniger Zerfall der Zellleiber anzuschliessen. Sie vergehen ebenso schnell, wie sie entstanden sind.

Auf welche Weise und auf welchem Wege wachsen nun diese zahlreichen Adenome weiter, wenn sie das ibnen durch das feste cirrbotiscbe Bindegewebe gesteckte Ziel erreicht haben? Oder wachsen sie daun überbaupt nicht weiter und bleiben auf diese Weise histologisch gutartige Geschwülste? Schon ibr rein zelliger Charakter, die ungewöhnliche Menge von Mitosen, die Neigung zu centralem Zerfall macht es aufs Höchste wahrscheinlich, dass ibnea die Tendenz zu maligner Entartung innewohnt. Schon makroskopisch war zu sehen, dass sie sich da, wo sie dicht liegen, gegeneinander abplatten, hier wird das Zwischengewebe, das also aus Leberresten und einem cirrhotischen Bindegewebsstrang besteht, auf ein Minimum zusammengedrängt, und dadurch entstebt eine Art Stroma, das die fast zusammenfliessenden Knoten in einzelne Theile zerlegt. Uebergangsbilder aller Art, sowie die Beobachtung von continuirlich durch diese Septa verlaufenden elastischen Faserzügen erweisen es mit Sicherheit, dass hier kein vom Tumor geilefertes Stroma vorliegt; das cirrhotische Bindegewebe bat die Function eines Tumor-Stromas äbernommen. Auch hier machen wir also wieder die Beobachtung, dass die Epithelzelle der einzig wesentliche Bestandtheil der epitbelialen Neubildung ist, dass sie in ihrer Weiterentwickelung für die Nabildung maassgebend 'ist und häufg genug, wie auch bier, ein fertiges Stroma vorfindet.

Hat nun ein Adenomknoten den ihm gebotenen Raum völlig ausgefüllt, und die Leberzellen zur völligen Druck-Atrophie gebracht, so beobachtet man in seiner Circumferenz häufig kleine Epithelstränge, die seiner Oberfläche parallel laufen. Ihre völlige Identität mit den Zellen der Hauptgeschwulst, besonders auch in Bezug auf die Farbenreaction, lässt keinen $Z$ weifel daräber, dass es sich um ein und dasselbe bandelt. Bewiesen wird dies durch directe brüclrenartige Zusammenbänge zwischen beiden. In dem Auftreten dieser Bilder erblicke ich in unserm Fall den ersten Schritt zur malignen Entartung der Adenome. Zwar überzengen wir uns sehr bald dass diese kleinen Tumorstränge nicht im cirrbotischen Bindegewebe liegen, sondern sie haben die einzelnen Lagen des druck-atrophischen Lebergewebes auseinander gedrängt, und sich in den alten Capillarspalten entwiekelt, deren Lumen sie eröffnen; daher ihre concentrisehe Anordnung zur Oberfläche der Muttergeschwulst. Meiner Auffassung könnte nun der Einwand gcracht werden, diese Dinge könnten von den dort zu Grunde gegangenen Leberzellresten ausgehen, die durch den Reiz der benachbarten Geschwulstbildung zu neuem Leben erwacht seien. Spräche biergegen schon der oben erwäbnte directe Zusammenbang, so fehlen auch vollständig die Uebergangsbilder von erwachenden Leberzellen zu Tumorzellen, oder gar eine Riesenzeilbildung. Nein! Hier hat der Tumor seinen Mutterboden veriassen. Uebrigens lässt sich das Fortwachsen in den Capillaren manchmal auch 
schon in der Peripherie kleinerer Knoten sehen, die noch allseitig von relativ wohl erbaltenem Parenchym begrenzt sind.

Sehr bald wachsen num die beschriebenen Tumorstränge zu grossen, auf dem Schnitt balbmondförmigen oder ringförmigen Gebilden aus, die ibre Muttergeschwulst umsehliessen. Hierbei sebeinen sie die offenbar schon gestörte Nahrungszufubr von ihrer Muttergeschwulst völlig abzuschneiden, und den hier sicherlich wohl vorhandenen Säftestrom ganz für ihr eigenes Wachstbum zu verwenden. Und so werden gerade die von den Tumorringen umschlossenen Adenomknoten bald nekrotisch, während die Ringe noch die beste Kernfärbung und Mitosenbildung aufweisen. In diesen Halbmonden und Ringen finden sich niemals Riesenzellen. Hat doch hier die Geschwulst ibren Mutterboden verlassen! Und so erblieke ich in dem constanten Fehlen von Riesenzellen in diesen walignen Theilen, die beste Bestätigung für meine Auffassung von diesen Riesenzellen. Die beschriebenen Balbmonde und Ringe hatte ich schon bei der makroskopischen Beschreibung kurz erwäbnt,

An ganz vereinzelten Stellen ist nun aber eine Aenderung des Geschwulstcharakters zu bemerken, die das Adenom dem Carcinom näher bringt: erstens liegen inmitten mancher Geschwulstknoten ganz besonders dicke Tumorstränge, sodass ein Uebergang in den alveolären Typus zu beginnen scheint; ferner siebt man das Centrum wohl gelegentlich durch einen structurlosen Zelibaufen gebildet, endlich aber ist es mir gelungen, eine ganz kleine Stelle zu finden, wo echter Krebs aus den Neubildungen hervorwächst, der schrankenlos in das cirrbotische Bindegewebe vordringt und keine Grenze mehr zu respectiren scheint. Ich habe makroskopisch die ganze eine Bälfte der Leber aû́ zabliosen Scbnitten auf ähnliche Stellen durchforscht, und habe mikroskopisch eine grosse Reihe von verschiedenen Stellen der Leber zum Theil auf grossen Schnitten durchmustert, und fühle mich daher za der Anscbaunng berechtigt, dass die Stellen, wo echter Krebs vorliegt, äusserst selten sind; icb babe nur diese eine gefunden. Ferner babe ich auch mikroskopisch die Gefässe verfolgt, und wieder ist es mir nur an einer einzigen Stelle gelungen, Geschwulstzellen in einem kleinen Gefässe, dessen Wand deutlich Muskelzellen enthielt, nachzuweisen; hier füllten sie das ganze Lnmen aus. Die Untersuchung von grösseren Gefässen, die von allen Seiten von Tumormassen bedrängt waren, ergab sonst stets, dass die Gefässwand von der Tumorwucherung respectirt wurde.

Damit bin ich mit der mikroskopischen Beschreibung zu Ende; sie ist so ausführlich geworden, weil ich zwischeadurch bereits die Deutung und einige kritische Betrachtungen eingeschaltet habe. Einiges muss ich jedoch nun noch folgen lassen. Ich weiss nicht, ob es einer ernstlichen Erörterung bedarf, dass auch hier in meinem Falle die Cirrhose die weitaus älteste von den Veränderungen ist. Alle Untersucher, die sich mit dieser 
Frage beschäftigt haben, halten die Cirrhose für älter, als die Adenom-Bildung, gleichviel ob sie einen Zusammenhang zwischen beiden für möglich halten oder nicht. Auch ist schon mehr als einmal constatirt worden, dass wenn irgend ein Zusammenhang besteht, die Cirrhose höchstens die Tumorbildung hervorgerufen haben könne, und nicht umgekehrt, weil sie diffus und gleichmässig entwickelt ist, und es eine ganze Anzahl von Stellen giebt, wo nur Cirrhose besteht, aber keine Geschwulstbildung. Meine mikroskopische Bischreibung hat wohl ebenfalls die Cirrhose als einen äusserst chronischen Process erscheinen lassen, die Tumorbildung aber als einen ganz jungen und schnell verlaufenden Vorgang. Wenn ich nun noch aus der Krankengeschichte wiederholen darf, dass seit mindestens zwei Jahren Verdacht auf Cirrhose bestand, und das Leiden bis zum Tode überhaupt nur unter den typischen Symptomen der Leber-Cirrhose verlief, dann glaube ich ihre Priorität für nachgewiesen halten zu dürfen. Wenn ich mir ein Urtheil darüber erlauben darf, wie lange vor dem Tode die ersten Anfänge der Adenombildung begonnen haben mögen, so möchte ich glauben, dass es sich wohl nur um ganz wenige Monate gehandelt haben mag; doch möchte ich das lediglich als eine Vermuthung betrachtet wissen.

Das Fehlen einer jeden Tumorbildung, besonders am Magen und Darmcanal, bestätigt unsere mikroskopisch völlig klar bewiesene Annahme, dass die Adenome in der Leber autochton entstanden, und nicht etwa als Metastasen aufzufassen sind, Metastasenbildung ist auch von unseren primären Lebertumoren noch nicht ausgegangen: nachdem der übrige Körper wenigstens theilweise durchsucht ist, und ich selbst noch die LeberhilusLymphknoten angesehen habe, scheint dies nicht der Fall zu sein. Freilich ist es schade, dass wir keine Nachricht über die Lungen haben; aber auch von den mannigfaltigen Geschwülsten der Leber ist mir keine einzige zu Gesicht gekommen, die als Metastase aufgefasst werden könnte. Entweder sind sie an Ort und Stelle entstanden, oder continuirlich fortgewachsen.

Ehe ich nun abschliessend behaupten kann, dass im Anschluss an die primäre Leber-Cirrhose eine vicariirende Hypertrophie, aus dieser Adenombildung, aus dieser endlich die maligne Entartung hervorgegangen sei, und ehe ich somit das Ganze 
als eine Kette consecutiver Vorgänge betrachten kann, muss ich einem letzten Einwande begegnen: Ist denn auch das an den Leberzellen constatirte Wachsthum überhaupt eine RegenerationsErscheinung oder nicht? Wenn nicht, dann ist an dieser Stelle die Kette der Ereignisse unterbrochen und der Zusammenhang zwischen Cirrhose und multipler Adenombildung ist weniger leicht verständlich, als sonst, und wir müssen auf die sonst $z u$ Hülfe herbeigezogenen ätiologischen Momente aus unserer Literatur zur Erklärung zurückgreifen; wir finden da Gallenstauung genannt, also ein chemisches Agens, und Druck von Seiten der Cirrhose, also ein mechanisches.

Es ist von vornherein äusserst wahrscheinlich, dass wir in der beschriebenen Hypertrophie der Leberzellen einen vicariirenden Vorgang vor uns haben; denn an vielen Stellen, wo von Tumorbildung noch gar keine Rede ist, sieht man sie in gleichmässiger Weise über das ganze Organ vertheilt an den noch am besten erhaltenen Parenchym-Zellen auftreten, und die Analogie mit zahllosen ähnlichen Fällen, wo dieselbe Hypertrophie ohne Tumorbildung vorliegt, lässt für sie eigentlich gar keine andere Deutung zu. Leider ist die Regeneration der Leber bei Cirrhose ein verhältnissmässig noch wenig bearbeitetes Gebiet. Aschoff sagt am Schlusse seines Berichtes über Epithel-Regeneration: „Die Untersuchungen über ächte Regeneration an cirrhotischer Leber sind zu gering, um ein abschliessendes Urtheil zu gestatten. Nach den bisherigen Erfahrungen muss die compensatorische Hypertrophie als die vor Allem in Betracht kommende Form des Wiederersatzes functionirenden Lebergewebes bei den chronischen Atrophien betrachtet werden". Daher habe ich es mir zur Aufgabe gemacht, den fraglichen Vorgang mit verwandten hypertrophischen und regenerativen Processen, die am Lebergewebe genauer beobachtet sind, zu vergleichen, und fand eine ganz auffallende, ja häufig in Einzelheiten ganz frappante Uebereinstimmung mit den Vorgängen in unserem Falle. Diese Uebereinstimmung ist meiner Auffassung nach um so überraschender, als der Mutterboden hier so sehr schwer verändert ist; ist doch kaum eine völlig normale Leberzelle mehr vorhanden, und doch so ähnliche Bilder!

Also ein abschliessendes Urtheil über Leber-Regeneration 


\section{4}

bei Cirrhose ist noch nicht zu fällen; dagegen existiren zahlreiche, zum Theil experimentelle Untersuchungen, über die Regeneration bei den acuten Atrophien (acute Leber-Atrophie, Verletzungen und Wiederersatz bei Entfernung ganzer Leberabschnitte). Aus diesen Untersuchungen gewinnt man den Eindruck, dass die Hauptquelle der unstreitig vorhandenen, echten Leber-Regeneration hier in den Gallengängen zu suchen sei und dass sich hier normal functionirendes, secernirendes Leber-Parenchym nach embryonalem Typus wiederbildet. Dem gegenüber scheint bei den chronischen Atrophien, Cirrhose, Staungs-Atrophie u. s. w. ein anderer Typus im Vordergrunde zu stehen; der Körper sucht den Verlust dadurch auszugleichen, dass die vorhandenen Leberzellen sich vergrössern und dadurch eine vermehrte Leistungsfähigkeit der einzelnen Zelle ermöglichen. Das ist wohl eine Ersatzwucherung, ist aber keine echte Regeneration, sondern eine vicariirende Hypertrophie, und tritt auch eine Vermehrung der Zellenzahl hinzu, so kann man von einer vicariirenden Hyperplasie sprechen. So entstehen die hypertrophischen Knoten in cirrhotischen und staungs-atrophischen Lebern. Mit dieser, wenu man so sagen darf, nothdürftigen Ersatzleistung scheint der Organismus dann wieder eine Zeit lang den Kampf gegen das Grundleiden weiterführen zu können; aber es scheint das letzte Aufgebot der Kräfte erfolgt zu sein; und während echte Regeneration vergeblich auf sich warten lässt, kann es, wie in unserem Falle, passiren, dass die mehr und mehr zunehmende Hypertrophie, gewissermaassen durch Ueberbürdung einzelner Elemente, durch einen unbekannten Reiz veranlasst, plötzlich in atypische Wucherung umschlägt, die wohl an embryonale Leberbildung erinnern kann, aus der jedoch nie fertig differencirtes, brauchbares Parenchym wird, und die sehr bald den letzten Rest leistungfähiger Substanz, der sie ihren Ursprung verdankt, auch noch za Grunde richtet.

Die erste grundlegende Arbeit über die Regeneration des Lebergewebes stammt von W. v. Podwyssozki jun. Es ist der erste, der echte Regeneration nachgewiesen hat. Nachdem er festgestellt hat, dass diese erstens von den Leberzellen selbst ausgehen kann, bespricht er zweitens die Regeneration, die von den Gallengängen ihren Ausgang nimmt. Jede dritte Möglich- 
keit weist er energisch von der Hand: "Eine Umwandlung von Bindegewebs-Elementen oder anderen Zellen nicht epithelialer Natur, z. B. Leukocyten (Griffini) in Leberzellen findet nicht statt; derartige Darstellungen beruhen entweder auf mangelhaften Untersuchungs-Methoden oder auf Beobachtungsfehlern." Seine Untersuchungen sind experimenteller Natur; er legt Verletzungen an, Stiche, Schnitte, Quetschungen und Extirpationen and beobacbtet nun den Regenerations-Typus und -Verlauf. Bei seinen Versuchsthieren überwiegt bei Ratten und Katzen die ausgehende Regeneration, von den Leberzellen selbst, bei Kaninchen und Meerschweinchen die von den Gallengängen. Uns interessirt hier natürlich hauptsächlich der erste Typus. Entsprechend seinen acut einsetzenden Krankheits- und Regenerations-Ursachen hat er natürlich einen sehr schnellen Verlauf beobachten können; aber auch bei ihm beginnt die Regeneration mit einer Vermehrung des Kern-Chromatins and eigenthümlicher Anordaung desselben, und alsbald folgt eine Vergrösserung des Kernes and der Zelle. Entsprechend dem schnellen und wirklich zur Zellneubildung führenden Verlauf beginnt nun eine lebhafte Mitosenbildung, und auch im einzelnen erinnern Podwyssozki's Bilder manchmal sehr an die von mir als Riesen-Mitosen beschriebenen Dinge (Fig. I, III, IV), insbesondere hat auch er gerade hier häufig Dreitheilungen beobachtet. Auch ähnliche Riesenzellen sah er, freilich nur, soweit ich verstehe, bei der von den Gallengängen ausgehenden Regeneration. Er fasst sie als zusammengeflossene Zellen auf, die sich dann später zu GallengangsEpithelien differenziren, letzteres hat er auch abgebildet. Auch er setzt den beschriebenen Vorgang mit der normalen Leberentwickelung in Parallele.

Somit erinnert unsere Hypertrophie aufs Lebhafteste an jene Vorgänge, nur zur Mitosenbildung kommt es in unserem Fall sehr viel seltener. Diese wenigen grossen Mitosen, die mir zu Gesicht gekommen sind, die scheinbar relativ häufig auch noch abnorm verlaufen, konnten uicht ausreichen, um ein abschliessendes Urtheil über sie zu gewinnen. Und doch zögere ich keinen Augenblick, in ihnen wenigstens den Versuch zu einer wirklichen Regeneration zu erblicken. Leider habe ich nicht genau verfolgen können, was aus ihnen wird, und was für Producte sie 
liefern; nur so viel kann ich mit Sicherheit annehmen, dass sie sich nicht an der Bildung der Adenomzellen betheiligen. Zur Adenom-Bildung werden diejenigen mehrkernigen Riesenzellen offenbar in erster Linie verwendet, deren Kern sich amitotisch getheilt hat, und ich weiss in der That nicht, $o b$ ich diese grossen Protoplasmamengen eigentlich schon zum Adenom oder noch zu seinem Mutterboden zählen soll; es ist eben die Uebergangsform. Dagegen bleibt bei manchen Riesenzellen die amitotische Kerntheilung aus, und der Kern wächst zu den geschilderten Riesenformen heran. Von ihnen gilt das, was Podwyssozki über die Vorbereitung zur Karyomitose sagt: „Die Ursache der Aufnahme assimilirten Materials in die Organisation des Kernes behufs Einleitung der Theilungsvorgänge entzieht sich der Erkenntniss", und weiter heisst es: "Bleibt trotz Vermehrung des Chromatins eine Karyomitose aus, so muss man annehmen, dass zwar günstige Ernährungsbedingungen gegeben waren, dass aber das Agens, welches den Anstoss zur Karyomitose zu geben vermag, nicht gebildet, oder nach seiner Entstehung sofort wieder zerstört oder an seiner Wirkung verhindert wurde“. Die riesenkernbergenden Riesenzellen scheinen grösstentheils einer weiteren Metamorphose nicht mehr fähig zu sein; denn gerade sie liegen noch lange, oft genug ganz vereinzelt, mitten in den fertigen Adenomen, manchmal ist ihr Kern abgeblasst, manchmal hat er sich in zwei auch noch riesenhafte Kerne zerlegt (Fig. VIII), manchmal aber, und das ist sehr merkwürdig, bilden sich mitosenartige Gruppirungen des Chromatins, die aber durchweg atypisch, pathologisch (?) sind und die wunderlichsten Bilder darbieten können. Entweder theilt sich ihr Chromatin in zahlreiche Klumpen, oder es bilden sich Tochtersterne, die ganz unregelmässig zu einander liegen. Neben diesen Tochtersternen bleiben dann oft noch formlose Massen zurück, die matter gefärbt sind; es scheint, als ob der Kern eine ganze Menge nicht mehr zur Mitose verwendbaren Materials aufgenommen habe (Fig. V). In Uebereinstimmung mit meinen vorhergehenden Schilderungen kann ich diese Mitosenbildung nur als einen letzten Versuch echter Leberzell-Regeneration auffassen; mit der Adenom-Bildung scheinen sie jedenfalls Nichts zu thun zu haben. Allein es sind Bilder dieser Art in unserem ganzen Präparat äusserst selten 
and schwer aufzufinden, und es muss bei ihnen die Frage offen gehalten werden, ob ihnen nicht eine ganz andere Bedeutung zukommt.

Die vicariirende Hypertrophie von Leberzellen bei chronischer Atrophie des Parenchyms durch Staung hat unter anderen im Göttinger Pathologischen Institut Herr Hainski an einem Fall von Lebervenen-Obliteration beschrieben. In seinem Falle kam es ebenfalls nu atypischer Wucherung and Bildung von tabulös gebauten Partien, also zu beginnender Adenom-Bildung, auf die er freilicb nicht sehr ausführlich eingeht.

Eine weitere, hierher gehörige Arbeit stammt von Meder. Er hat die Regeneration bei acuter Leberatrophie an einer Reihe von Fällen studirt und beschrieben. Doch es würde im Rahmen dieser Betrachtungen zu weit führen, wollte ich auf all' diese Arbeiten näher eingehen. Meder giebt über die Literatur eine kritische Zusammenstellung. Er resumirt: „Eine Regeneration duch Proliferation der noch erhalten gebliebenen Leberzellen ..... ist mit Sicherheit nachzuweisen. Diese Regeneration würde sich ganz im Rahmen des Normalen abspielen ohne wesentliche Veränderung der Leberstructur." Was den Vorgang an den Zellen selbst anbetrifft, so sagt er: "Die noch erhaltenen Leberzellen nehmen an Zahl und Grösse inverhalb der normalen Anordnung zu. Dadurch vergrössern sich natürlich zugleich die Leberzellbälkchen und Acini zwecks Ersatzes des verloren gegangenen Parenchyms. Es handelt sich also hier um eine Art vicariirender Hypertrophie, ähnlich wie sie, allerdings in noch viel erheblicherem Maasse statt hat bei Leber-Exstirpationen und bei Lebertumoren, Echinococcus, ferner bei der sogenannten knotigen Hyperplasie der Leber bei Cirrhose, gelappter Leber bei Syphilis und Staungsleber," Nachdem er dann noch festgestellt hat, dass bei der acuten Leber-Atrophie die Regeneration von den Gallengängen aus überwiegt, schildert er diese, und spricht dabei auch von „riesenzellartigen Uebergangsformen von gewucherten Gallengangs-Epithelien zu neugebildeten Leberzellen." Marchand constatirt drei Arten von Leber-Regeneration bei acuter Leber-Atrophie:

1) Hyperplastische Wucherung grösserer, erhalten gebliebener Reste von Drüsenparenchym. 
2) Wucherung isolirter Leberzelireste im Bereiche des stärksten Zerfalls.

3) Wucherung der interacinösen Gallengänge.

Wenn es somit als feststehend betrachtet werden kann, dass es Wiederersatz functionirender Leberzellen von den übrigbleibenden aus giebt, so haben wir alle Veranlassung, anzunehmen, dass dieser Typus bei den chronischen Atrophien, insbesondere auch bei der Lebercirrhose zum mindesten überwiegt, und in einer vicariirenden Hypertrophie und Hyperplasie ihren Ausdruck findet. Das liegt auch in unserem Falle vor; und wenn somit die Kette der Veränderungen sich zwanglos zu einem Ganzen fügt, wenn die Cirrhose als der zweifellos älteste Vorgang die vicariirende Hypertrophie, diese wiederum die Adenom-Bildung hervorgerufen hat, so ist nur noch ein Wort bier am Platze über den malignen Charakter unserer Adenome, um damit zu schliessen, dass hier echter Krebs autochton in der Leber entstanden, und jedenfalls indirect durch die primäre Cirrhose hervorgerufen ist. Diesen innigen Zusammenhang beeinträchtigt natürlich keineswegs die Thatsache, dass wir einen unbekannten Reiz (chemischer, infectiöser, mechanischer, nutritiver Art, in der literatur fehlt es ja vicht an Hypothesen derart) annehmen müssen, der in diesem Falle diffus einwirkend die "Metatypie" (Rindfleisch) der Wucherung hervorrufen half. Was die Malignität unsrer Adenume anbetrifft, so wird, denke ich, die gegebene mikroskopische Beschreibung zum Beweise genügen; zwar ist das schrankenlose Wachsthum, das Durchbrechen gegebener Grenzen, insbesondere das Hineinwachsen ins Bindegewebe vorläufig nur an wenigen Stellen nachzuweisen. Die Hauptmasse der Tumoren bat zwar äusserst schnell wachsenden Charakter, liegt aber noch im Rahmen des Gutartigen. Und so ist denn die terminale Verschlimmerung und der Tod nicht auf Rechnung der malignen Entartung zu setzen, sondern auf Rechnung der ersten, noch nicht ausgesprochen malignen Adenom-Bildung, die den letzten Rest functionirenden Leberparenchyms vernichtete.

Frohmann ist nun durch eine Beobachtung zu der Ueberzeugung gelangt, dass die Cirrhose und die Adenom-Bildung aichts mit einander zu thun hätten; er fand: "dass gerade die am stärksten hypertrophischen Partien der Leber von der Geschwulst- 
Entartung verschont geblieben sind." "Ich halte dies einerseits deswegen nicht für beweisend, weil ja auch ich gefunden habe, dass die besonders lebhaft hypertophischen Leberzellen auf den Neubildungsreiz nicht mehr reagiren; andererseits frage ich mich, warum soll denn nicht ein grosser Theil einfach hypertrophisch bleiben?: Müssen sie denn alle adenomatös entarten? Vielleicht wandeln sie sich auch noch später in Adenom um, wenn das Leben länger fortbesteht. Bei dieser Auswahl spielen ganz fraglos noch eine Menge von Factoren mit, besonders denke ich hier an Circulations- und Secretions-Verhältnisse, an den chemischen Aufbau der Zell- und Kernsubstanz u. s. w., die wir vielleicht in ihrer Wirkung und Wechselwirkung auf einander niemals völlig ergründen können. Ausserdem haben ich selbst und Audere, besonders auch Siegenbeek van Heukelom Uebergangsbilder von hypertrophischen Leberzellen in Tumorzellen gesehen.

Die Cirrhose ist das erste in der Kette der ätiologischen Momente, ohne sie wäre in diesem Falle die multiple AdenomBildung fraglos ausgeblieben; das ist es, was mich veranlasst, Simmonds in seiner Eintheilung beizustimmen. Die diffuse ältere Veränderung wird zum ätiologischen Momente und giebt damit die Berechtigung, die multiplen Adenome der Leber als eine besondere Gruppe abzusondern.

Es ist nun ja keineswegs wahrscheinlich, dass sich die Tumorbildung in unserem Falle mit der Geschwulst-Genese im Allgemeinen in Parallele setzen liesse, geschweige denn, dass sie als Typus aufgestellt werden kann. Sollte aber der Vorgang derselbe sein, so könnten wir aus diesen Bildern die Anschauung gewinnen, dass die Tumorbildung mit einer Hypertrophie normaler Zellen, die in diesem Falle dem Organismus auch in normaler Weise eingefïgt waren, beginuen kann. $\mathrm{Zu}$ dieser Hypertrophie ist natürlich eine Veranlassung erforderlich; diese wird hier durch einen Parenchym-Verlust gegeben. Diesem Agens muss sich ein zweiter, unbekannter Reiz zugesellen, auf dessen Rechnung dann die Metatypie sowohl, als anch die enorme Wachsthums-Energie zu setzen sein würde. Jedem, der schon einmal über Geschwulst-Genese nachgedacht hat, muss ein solcher Fall in diese Gedanken-Richtung führen, wo man jedoch sehr bald auf Schritt und Tritt ungelösten Problemen begegnet. Nirgends 
aber lässt sich jedenfalls die Entstehung von primären epithelialen Neubildungen so genau und in so jungen Anfängen verfolgen, als bei den multiplen malignen Leber-Adenomen.

Mein verehrter Chef, Herr Geheimrath Orth, hat mir die vorstehenden Untersuchungen übertragen und mir bei ihrer Ausführung geholfen. Es sei mir daher gestattet, ihm auch an dieser Stelle meinen aufrichtigen Dank zu sagen.

\section{Erklärung der Abbildungen auf Taf. VIII.}

Fig. 1. Hypertrophische Leberzelle, Riesenzelle, in beginnender indirecter Kerntheilung.

Fig. II. Hypertrophische Leberzelle, Riesenzelle, mit vacuolen-ähnlichen Bildungen im Innern des Riesenkernes. (Beginnende amitotische Theilung?)

Fig. III. Riesen-Mitose in einer bypertrophischen Leberzelle.

Fig. IV. Riesen-Mitose in einer hypertrophischen Leberzelle (Triaster).

Fig. V. Riesenzelle, die vereinzelt mitten im Adenomgewebe liegt. Vjelfache mitosenartige Gruppirungen des Chromatins mit einem heller gefärbten Rückstande.

Fig. VI. Uebergang der hypertrophischen Leberzellen in Adenom. In der Uebergangszone deutliche Zellhypertrophie und Bildung von Riesenzellen mit Riesenkronen.

Fig. VII. Kleiner Adenomknoten wit dem angrenzenden hypertrophisehen Lebergewebe, aus dem er hervorgegangen, Compression der anliegenden Theile durch das zunehmende Wachsthum.

Fig. VIII. Isolirt im Geschwulstgewebe liegengebliebene Riesenzelle mit einem getheilten Riesenkerne.

\section{Alphabetisches Literaturverzeichniss.}

Asch off: Referat überRegeneration. Lubarsch-Ostertag; Ergebnisse 1898.

R. Beneke: Zieglers Beiträge, IX S. 484.

Birch-Hirschfeld: Pathologische Anatomie, 1877, S. 952.

Ebertb: Untersuchungen über die normale und patbologische Leber. 3) Das Adenom der Leber. Dieses Archiv, 1868, Bd. 43, S. 1-8.

Friedreich: Ueber multiple knotige Hyperplasie der Leber und Milz. Dieses Arch., 1865. Bd. 33, S. $48-65$ und S. 553.

Frohmann: Ueber das Leber-Adenom nebst Bemerkungen über 'Theilungsvorgänge an den Leberzellen. Inaug. Diss. Königsberg, Juli 94.

Greenisb: Ueber das Adenom der Leber. Wien. med. Jahrb., 1882, S. 411. Griesinger: Das Adenoid der Leber. Archiv für Heilkunde, 1864, S. 385 bis 394. 
Hainski: Ein Fall von Lebervenen-Obliteration. Inaug. Diss. Helmstaedt, 1884. (Aus dem Pathologischen Institut in Göttingen.)

Hansemann: Ueber den primären Krebs der Leber. Berl. klin. Wochenschrift, 1890, No. 16, S. 353.

Hoffmann: Grosses Adenom der Leber. Dieses Arch., 1867, Bd. 39, S. 193. Homann: Ein Fall von Leber-Adenom. Inaug. Diss. Würzburg, 1888.

Jungmann: Ein Fall von cirrbotischer Leber mit Adenom-Bildung und Uebergang dess. in Carcinom. Inaug. Diss. Berlin, 1881. (Aus dem Pathologischen Institut in Göttingen.)

Klebs: Handbuch der pathologischen Anatomie, I, 1819, S. 375.

Klob: Zur pathologischen Anatomie der Leber. Fall von acuter, gelber Leber-Atrophie, scheinbare Leber-Adenoide. Wiener med. Wochenschrift, 1865, No. $75,76,77$.

Marchand: Ceber den Ausgang der acuten Leberatrophie in multiple knotige Hyperplasie. Ziegler, Beiträge XVII, S. 206.

Markwald: Das multiple Adenom der Leber. Dieses Arch., Bd, 144, S. 29.

Meder: Ueber acute Leber-Atrophie mit besonderer Berücksichtigung der dabei beobachteten Regenerations-Erscheinungen. Zieglers Beiträge XVII, S. 143 .

Orth: Pathologische Anatomie, 1887, S. 955.

Pawlowski: Zur Lehre von den Adeuomen der Leber, Tubulöses cavernöses Adenom. St. Petersburger med. Wochenschrift, 1884, No. 8, S. 73.

Perls: Lehrbuch der allgemeinen path. Anathomie und Pathogenese, 1877, Bd. I, S. 48 i.

v. Podwyssozki: Experimentelle Untersuchungen über die Regeneration der Drüsengewebe. Zieglers Beiträge I, S. 259.

Rindfleisch: Mikroskopische Studien über das Leber-Adenoid. Archiv für Heilkunde, 1864, S. 394-401.

Rokitansky: 1) Wiener allgem. medicinische Zeitschr, 1859, No. 14.

2) Lehrbuch der patb. Anatomie. Bd. III, 1861, 3. Aufl.

Siegenbeek van Heukelom: Das Adenocarcinom der Leber mit Cirrhose. Zieglers Beiträge, XVI, S. 341 .

Simmonds: Die knotige Hyperplasie und das Adenom der Leber. Archiv für klinische Medicin. Bd. 34, 1884, S. 388 .

Staats: Ein Fall von Adenoma bepatis. Inaug. Diss. Lippstadt, 1886. Stroebe: Bericht über die Verhandlungen der Section für allgem. Pathologie und path. Anatomie auf der 66. Versammlung deutscher Naturforscher und Aerzte zu Wien, 24.-30. September 1894. Centralblatt für allgem. Pathologie und path. Anatomie 5, 1894, S. 846.

Wagner: 1) Die Structur des Leberkrebses. Archiv für Heilkunde, 1861, S. 209. 2) Drüsengeschwult der Leber. Arch. f. Heilk., 1861, S. 473.

Willigk: Beitrag zur Histogenese des Leber-Adenoms. Dieses Arch., 1870, Bd. 51, S. 208.

Wulff: Der primäre Leberkrebs. Inaug. Diss. Tübingen, 1876. 